\title{
Investigating Tunneling Controlled Chemical Reactions through ab-initio Ring Polymer Molecular Dynamics
}

\author{
Xinyang Li and Pengfei Huo* \\ Department of Chemistry, University of Rochester, 120 Trustee Road, Rochester, New York \\ 14627, United States \\ E-mail: pengfei.huo@rochester.edu
}

\section{Abstract}

We use the ab-initio ring polymer molecular dynamics (RPMD) approach to investigate tunneling controlled reactions in methylhydroxycarbene. Nuclear tunneling effects enable molecules to overcome the barriers which can not be overcome classically. Under low-temperature conditions, intrinsic quantum tunneling effects can facilitate the chemical reaction in a pathway that is neither favored thermodynamically nor kinetically. This behavior is referred to as the tunneling controlled chemical reaction and regarded as the third paradigm of chemical reaction controls. In this work, we use the $a b$-initio RPMD approach to incorporate the tunneling effects in our quantum dynamics simulations. The reaction kinetics of two competitive reaction pathways at various temperatures are investigated with the Kohn-Sham density functional theory (KS-DFT) on-the-fly molecular dynamics simulations and the ring polymer quantization of the nuclei. The reaction rate constants obtained here agree extremely well with the experimentally measured rates. We demonstrate the feasibility of using $a b$-initio RPMD rate calculations in a realistic molecular system, and provide an interesting and important example for future investigations on reaction mechanisms dominated by quantum tunneling effects.

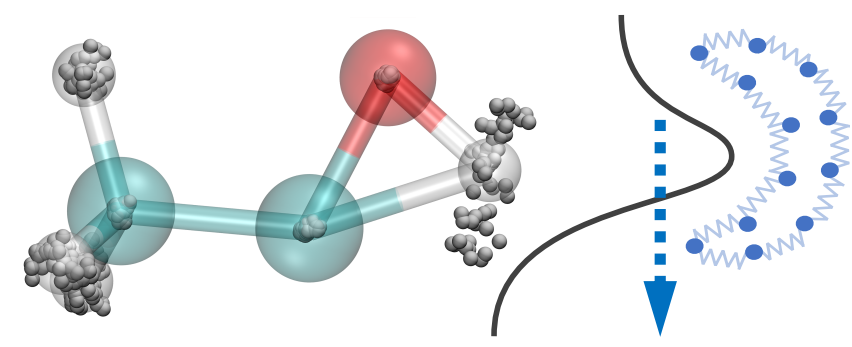

Nuclear quantum effects (NQEs), such as quantum tunneling and zero-point energy (ZPE), have shown to play a crucial role in various chemical processes, ${ }^{1,2}$ ranging from hydrogen bonding, ${ }^{3-7}$ proton transfer reactions, ${ }^{8-11}$ hydride transfer reactions, ${ }^{12,13}$ proton-coupled electron transfer (PCET) reactions ${ }^{14-17}$ and catalytic reactions. ${ }^{18-21}$

NQEs enable molecules to directly tunnel through the potential energy barriers that are otherwise formidably high classically. ${ }^{22}$ Although the importance of tunneling effects in chemistry has long been acknowledged, ${ }^{23}$ the attention on the ability of actively using tunneling to control chemical reaction directions only starts to emerge recently. ${ }^{24-26}$ Tunneling control refers to the scenarios that a kinetically or thermodynamically less favored reaction pathway becomes the dominating one due to the reaction enhancement by intrinsic quantum tunneling effects. ${ }^{24-26}$ This phenomenon is recently regarded as the third paradigm of chemical reaction control, beyond the traditional thermodynamic and kinetic controls. ${ }^{26}$

Methylhydroxycarbene (MHC), a hydroxycarbene derivative, has emerged as an excellent example of the tunneling controlled reactions. ${ }^{24,27}$ There are two hydrogen atom transfer pathways to the divalent carbon atom in MHC, illustrated in Fig. 1. We denote reaction pathway $\mathrm{A}$ as the hydrogen atom transfer from the methyl group to the carbine center, forming vinyl alcohol; and reaction pathway $\mathrm{B}$ as the transfer of the hydrogen atom in the hydroxy group to the carbine center, resulting in acetaldehyde. Both reactions causes the decay of the MHC molecule. At low temperatures, the decay of $\mathrm{MHC}$ results in acetaldehyde through reaction $\mathrm{B}$, forming a product that is favored nei- 
ther kinetically nor thermodynamically. This leads to a completely tunneling controlled product. ${ }^{24-26}$ Many more recent examples of the tunneling controlled reactions can be found in a recent review in Ref. 26. Accurately simulating the tunneling controlled chemical reaction requires the explicitly description of the NQEs, which is beyond the classical rate constant theory or classical molecular dynamics simulation.

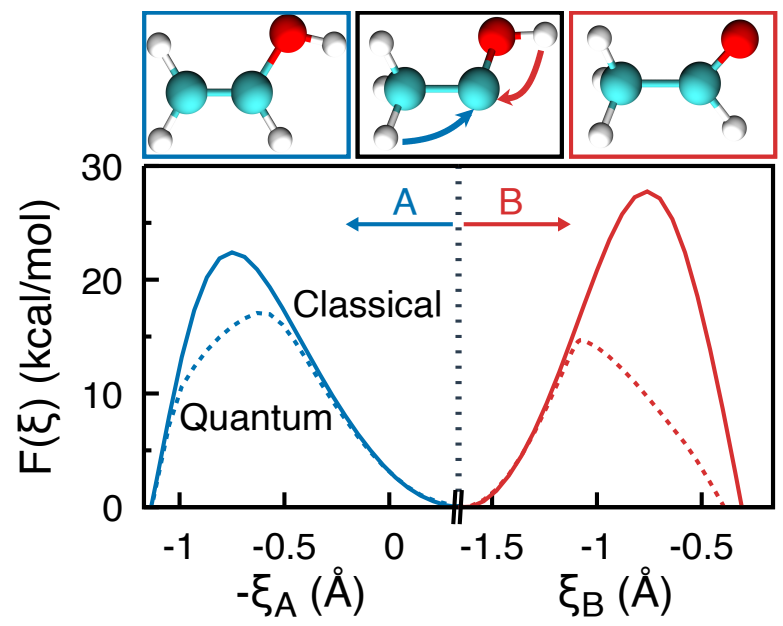

Figure 1: The classical (solid lines) and quantum (dashed lines) free energy profiles focused in the barrier region at $200 \mathrm{~K}$ for both reaction pathway $\mathrm{A}$ (blue) and B (red). The geometries in the top panel correspond to vinyl alcohol (product A), methylhydroxycarbene (reactant) and acetaldehyde (product B), respectively. The arrows shows the transferring direction of the hydrogen atoms. Note that to indicate the HAT direction of reaction $\mathrm{A},-\xi_{A}$ is used as the $\mathrm{x}$-axis in this plot.

In this work, we use the ring polymer molecular dynamics (RPMD) ${ }^{11,28-31}$ to compute the reaction rate constants for two competing reaction paths in the MHC molecule. RPMD is an approximate quantum dynamics approach based on Feynman's imaginary-time path-integral formalism, ${ }^{32}$ which provides exact quantum statistics and approximate yet accurate quantum dynamics. ${ }^{28,33,34}$ In this formalism, each atom is represented by a ring polymer of $n$ beads (imaginary-time slices), with a harmonic spring connecting the adjacent beads. ${ }^{35}$

Quantum reaction rate constants can be accurately evaluated by using the RPMD fluxside correlation function formalism, which has been extensively discussed in the previous literature. ${ }^{12,14,29-31,36}$ Here, we demonstrate perhaps the first ab-initio RPMD rate constant calculation. We combine RPMD with ab-initio on-the-fly simulations at the level of Kohn-Sham DFT (with the BLYP functional ${ }^{37-39}$ and in its singlet state) using a plane-wave basis to investigate the competing hydrogen atom transfer (HAT) reactions in MHC. The computational details are provided in the Theoretical Approaches.

For a molecular system with $\mathcal{N}$ total nuclear degrees of freedom (DOF) (or $\frac{1}{3} \mathcal{N}$ total number of atoms), the corresponding ring polymer Hamiltonian is expressed as

$H_{n}(\mathbf{p}, \mathbf{q})=\sum_{j=1}^{n}\left[\frac{\mathbf{p}_{j}^{2}}{2 \mathbf{M}}+V\left(\mathbf{q}_{j}\right)+\frac{1}{2} \mathbf{M} \omega_{n}^{2}\left(\mathbf{q}_{j}-\mathbf{q}_{j-1}\right)^{2}\right]$

where $n$ is the total number of copies of the original system (beads), $\mathbf{q}_{j}=\left\{\left[q_{1}\right]_{j},\left[q_{2}\right]_{j}, . .,\left[q_{\mathcal{N}}\right]_{j}\right\}$ and $\mathbf{p}_{j}=\left\{\left[p_{1}\right]_{j},\left[p_{2}\right]_{j}, . .,\left[p_{\mathcal{N}}\right]_{j}\right\}$ are the position and momenta vector of the $j_{\text {th }}$ bead, with mass $\mathbf{M}=$ $\left\{M_{1}, M_{2}, . ., M_{\mathcal{N}}\right\}$. Further, $V\left(\mathbf{q}_{j}\right)$ is the adiabatic potential energy surface for the nuclei, and the inter-bead ring polymer frequency is $\omega_{n}=n / \beta \hbar$.

The approximate quantum mechanical rate constant is calculated as the plateau value of the RPMD flux-side correlation function. ${ }^{11,28,31}$ To facilitate the numerical simulations, we apply the Bennett-Chandler scheme ${ }^{40}$ that express the rate constant as follows ${ }^{11,14,36}$

$$
k=\lim _{t \rightarrow t_{\mathrm{p}}} \kappa(t) \cdot k_{\mathrm{TST}},
$$

where $t_{\mathrm{p}}$ refers to the plateau time of the flux-side correlation function, $k_{\mathrm{TST}}$ is the RPMD-TST rate constant, and $\kappa(t)$ is the transmission coefficient that captures the dynamical recrossing. The details of the $k_{\text {TST }}$ is provided in the Theoretical Approach, and $\kappa(t)$ is expressed as follows

$$
\kappa(t)=\frac{\left\langle\mathcal{F} \cdot h\left[\xi\left(\overline{\mathbf{q}}_{t}\right)-\xi^{\ddagger}\right]\right\rangle_{\mathrm{c}}}{\left\langle\mathcal{F} \cdot h\left[\dot{\xi}\left(\overline{\mathbf{q}}_{0}\right)\right]\right\rangle_{\mathrm{c}}},
$$

where $\overline{\mathbf{p}}=\frac{1}{n} \sum_{j} \mathbf{p}_{j}$ and $\overline{\mathbf{q}}=\frac{1}{n} \sum_{j} \mathbf{q}_{j}$ are the the centroid of the momenta and positions, respectively. The 0 and $t$ subscripts in $\mathbf{q}$ indicates the initial time and time $t$. In addition, $h$ is the side operator, which is a Heaviside function of the reaction coordinate $\xi$ that differentiate the reactant versus the product, and $\mathcal{F}$ is the flux operator which is the time derivative of the side operator $\mathcal{F}\left(\overline{\mathbf{q}}_{0}, \overline{\mathbf{p}}_{0}\right)=\dot{h}\left[\xi\left(\overline{\mathbf{q}}_{0}\right)\right]$. Further, $\langle\ldots\rangle_{\mathrm{c}}$ denotes the ensemble average over trajectories that are initially constrained on the dividing surface $\xi^{\ddagger}$. 
The transmission coefficient as well as the free energy profiles are evaluated with the ring polymer trajectories governed by the RPMD Hamiltonian $\hat{H}_{n}$ in Eq. 1, whereas the potential $V\left(\mathbf{q}_{j}\right)$ is evaluated through ab-initio on-the-fly simulation. The largest $\kappa(t)$ simulation of this work require the on-the-fly propagation of a $\frac{1}{3} \mathcal{N} \times n=7 * 64=$ 448 atoms fictitious molecular ring-polymer for up to $10^{3}$ trajectories, with at least 450 electronic structure calculations along every single ring polymer trajectory. Additional details of the numerical simulations are provided in Theoretical Approaches. Using the ab-initio RPMD approach combined with the enhanced sampling technique (the blue moon ensemble approach, ${ }^{41-43}$ see details in the Supporting Information), we can directly simulate the classical (when using $n=1$ ) and quantum mechanical free energy profiles (potential of mean force).

Fig. 1 presents the classical potential of mean force (solid curves) and the quantum potential of mean force (dashed curve) $F(\xi)$ (defined in Eq. 5) at $T=200 \mathrm{~K}$, along the reaction coordinate $\xi$ (defined in Eq. 4) for both reaction $\mathrm{A}$ (blue) and $\mathrm{B}$ (red). It is clear that the classical free energy barrier of pathway $\mathrm{B}$ is higher than reaction $\mathrm{A}$, but also thinner than pathway A. Compared to the classical free energy barrier, the quantum free energy barriers are significantly lower for both reaction pathways. Further, reaction B is now having an even lower free energy barrier than reaction A. This is because that the NQEs start to dominate the reaction mechanism below the the crossover temperatures ${ }^{44} T_{\mathrm{c}}=\frac{\hbar \omega^{\ddagger}}{2 \pi k_{\mathrm{B}}}$, where $\omega^{\ddagger}$ is the imaginary barrier frequency at the transition state (TS), and tunneling is more sensitive to the barrier width, rather than the potential barrier height. The crossover temperature were reported to be $321 \mathrm{~K}$ and $462 \mathrm{~K}$ for pathway $\mathrm{A}$ and $\mathrm{B}$, respectively. ${ }^{27}$ Since tunneling effects are essential to the reaction mechanism below the crossover temperature, this highlights the importance of using a method which includes tunneling effects in the simulations of this system.

Fig. 2A provides the typical configurations of molecular ring polymer along the the reaction coordinate of reaction $\mathrm{B}$. The labels for each configuration indicate a particular value of $\xi_{\mathrm{B}}$ in panel $\mathrm{B}$. At the transition state ensemble, the ring polymer spans over the barrier into the reactant and product sides. The reaction pathway B allows the most beads to reach to lower potential positions, due to a thinner potential barrier. Since the ring polymer radius is particularly large at lower temperatures (due to the lower $\omega_{n}$ in $H_{n}$ of Eq. 1), this effect plays an essential role at low temperatures.

Fig. 2B presents free energy profiles $F(\xi)$ at 200 $\mathrm{K}$ of reaction pathway $\mathrm{B}$, simulated with the classical $a b$-initio MD (AIMD) with $n=1$ (red) and the quantum RPMD with $n=32$ (blue). Apparently, without considering NQEs, classical AIMD overestimates the free energy barrier height by more than $10 \mathrm{kcal} / \mathrm{mol}$.

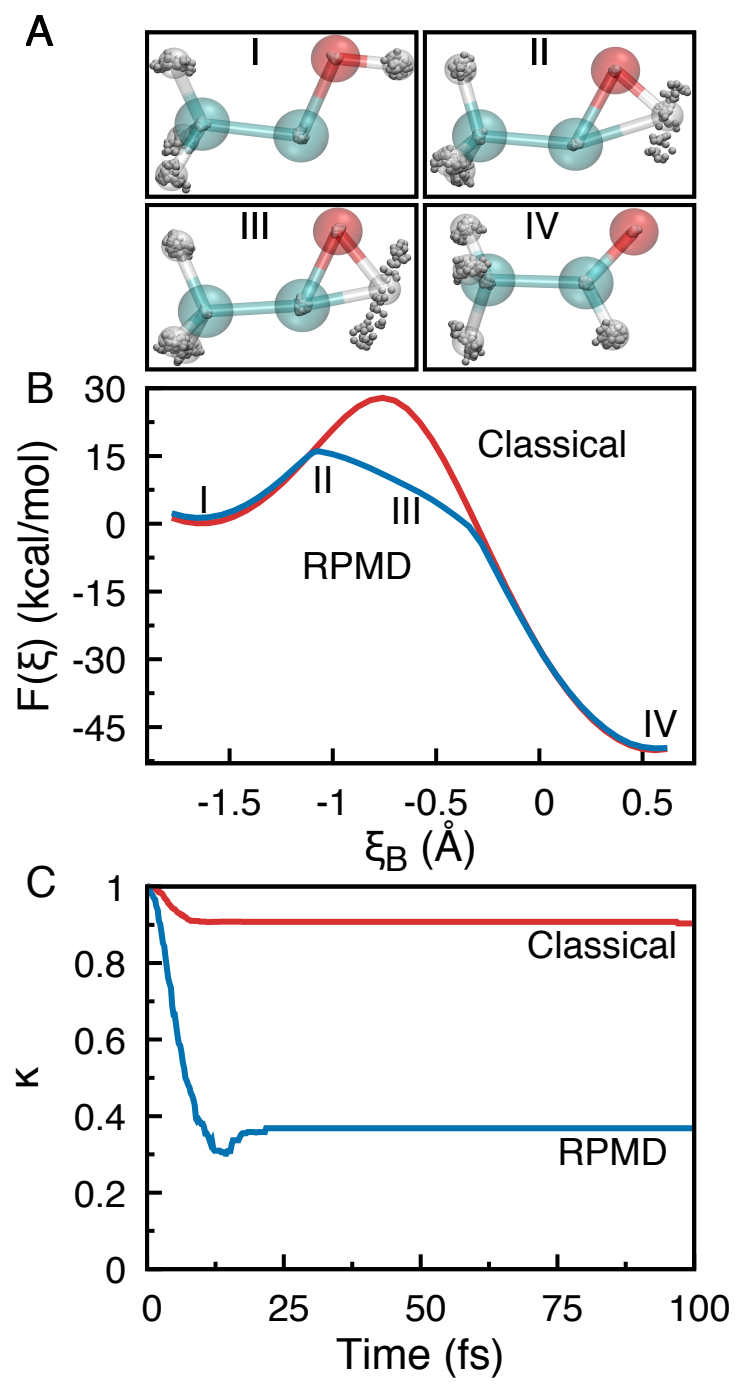

Figure 2: Simulation results for reaction pathway $\mathrm{B}$ at $200 \mathrm{~K}$. (A) The typical ring polymer configurations of the reactant (I), TS (II) and product (IV), as well as a configuration (III) between the TS and product. (B) The classical (red) and quantum (blue) free energy profiles $F(\xi)$. (C) The classical (red) and quantum (blue) transmission coefficient $\kappa(t)$.

After reaching the TS configuration (II in 
Fig. 2A), the molecular ring polymer goes into a "sliding downhill" process, as seems that it directly cut through the barrier. This is because at such a low temperature, the ring polymer is overstretched (III in Fig. 2A), and the top of the free energy barrier based on the centroid coordinate is no longer capable to present an optimized dividing surface that minimizes recrossing. ${ }^{20,44}$ We emphasize that this is a well-known feature of RPMD, which does not significantly influence the accuracy of the rate constant so long as the $\kappa(t)$ (which accounts for the recrossing) is also explicitly included in the rate constant. ${ }^{44,45}$

Fig. $2 \mathrm{C}$ presents the classical and quantum timedependent transmission coefficients $\kappa(t)$ at $200 \mathrm{~K}$ for the reaction pathway $\mathrm{B}$. The plateau value $\kappa\left(t_{\mathrm{p}}\right)$ is lower in the quantum simulation than the classical one, ${ }^{12,14}$ as the quantum free energy barrier is lower and the stretching of the ring polymer causes a "tug of war" scenario. ${ }^{20}$

To investigate the temperature dependence of the rate constant, we also perform the simulations at $T=400 \mathrm{~K}, 300 \mathrm{~K}$, and $120 \mathrm{~K}$ for reaction pathway B. Similarly, we computed these quantities for reaction pathway $\mathrm{A}$. These additional results are provided in the Supporting Information (Fig. S1). Fig. 3A presents the ring polymer transition state configurations at four temperatures. The radius of the ring polymer for all atoms increases as the temperature decreases, because the spring constant of the ring polymer becomes weaker $\left(\omega_{n} \propto T\right)$. As the temperature decreases, the ring polymer of the transferring hydrogen atom becomes more stretched on the top of the potential barrier, indicating a stronger tunneling effect.

Fig. 3B presents the temperature dependence of the free energy profile along the reaction coordinate $\xi_{\mathrm{B}}$. As we expected, the free energy barrier becomes lower at a lower temperature. When the temperature is below $\frac{1}{2} T_{\mathrm{c}}$ (half of the crossover temperature), there is a clear "edge cutting" behavior along the reaction coordinate, which is originated from evaluating the free energy profile with a centroid coordinate. ${ }^{20,44}$

Fig. 3C presents the transmission coefficient $\kappa(t)$ computed under four different temperatures. Not surprisingly, the plateau value $\kappa\left(t_{\mathrm{p}}\right)$ decreases at a lower temperature, due to lowered barriers and the complex motion of the over-stretched ring polymer in the intermediate time, leading to more recrossing events. The relatively low value of $\kappa\left(t_{\mathrm{p}}\right)$ at 200 $\mathrm{K}$ and below also clearly indicated that the top of the free energy barrier based on the centroid coordinate is no longer an optimized dividing surface that minimize recrossing. ${ }^{44,45}$

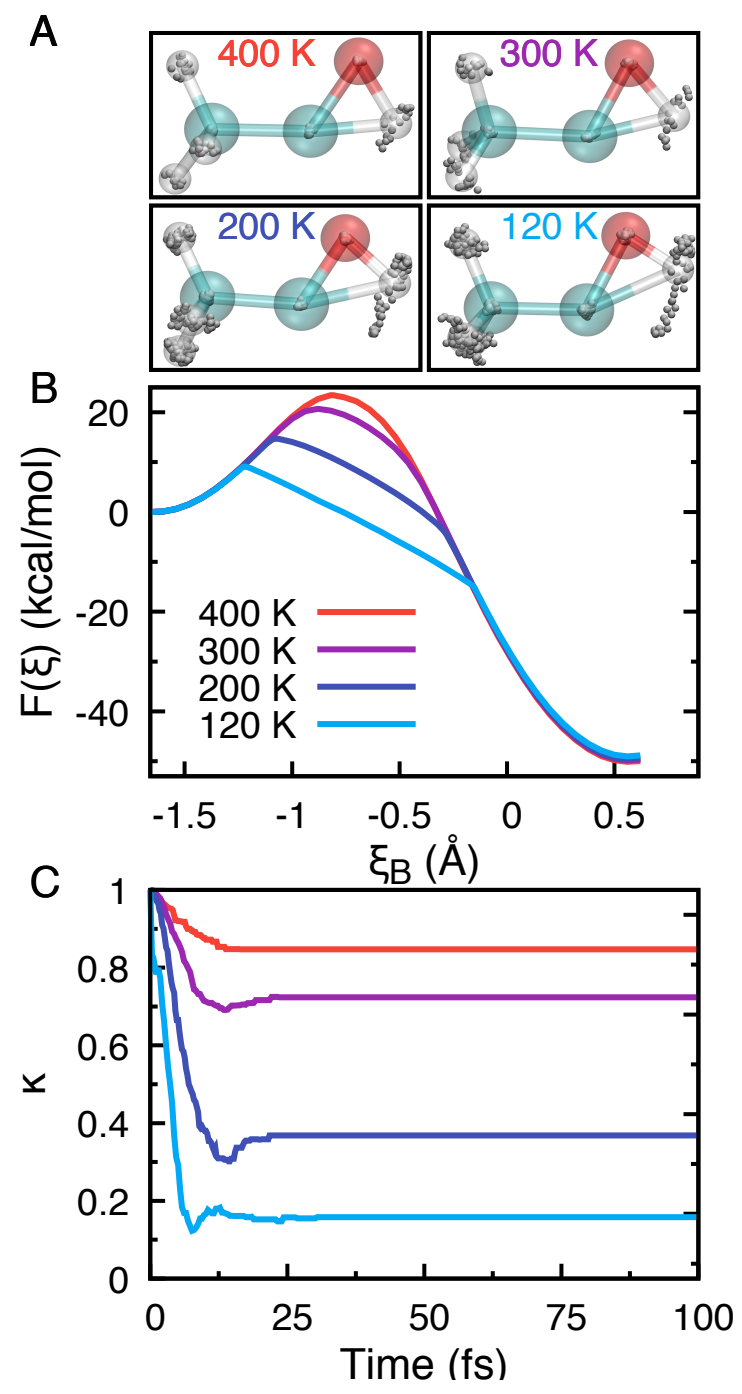

Figure 3: Temperature-dependence of of the reaction pathway B. (A) The representative ring polymer TS configurations at four different temperatures. (B) The quantum free energy profiles $F(\xi)$ at four temperatures corresponding to panel A. The free energy barrier $\Delta F\left(\xi^{\ddagger}\right)$ decreases as the temperature directions. (C) The time-dependent transmission coefficient $\kappa(t)$ plots at four different temperatures. The plateau value $\kappa\left(t_{\mathrm{p}}\right)$ decreases as the temperature decreases.

Fig. 4 presents the plots of the rate constant for the reaction pathway $\mathrm{A}$ (blue) and $\mathrm{B}$ (red), obtained by plugging $k_{\mathrm{TST}}$ computed from the free energy barrier heights and the plateau value of the transmission coefficients $\kappa\left(t_{\mathrm{p}}\right)$ into Eq. 2. There exists a crossover of the two rate constants in the 
temperature range between $200 \mathrm{~K}$ and $300 \mathrm{~K}$, indicating a switch in the preferred reaction mechanism. Under the high temperature limit, the free energy barrier of the reaction pathway $\mathrm{A}$ is much lower than the barrier of pathway B (by around $5 \mathrm{kcal} / \mathrm{mol}$ at $T=400 \mathrm{~K}$ ). Consequently, the reaction rate of pathway $\mathrm{A}$ is higher in this case, making vinyl alcohol the preferred product.

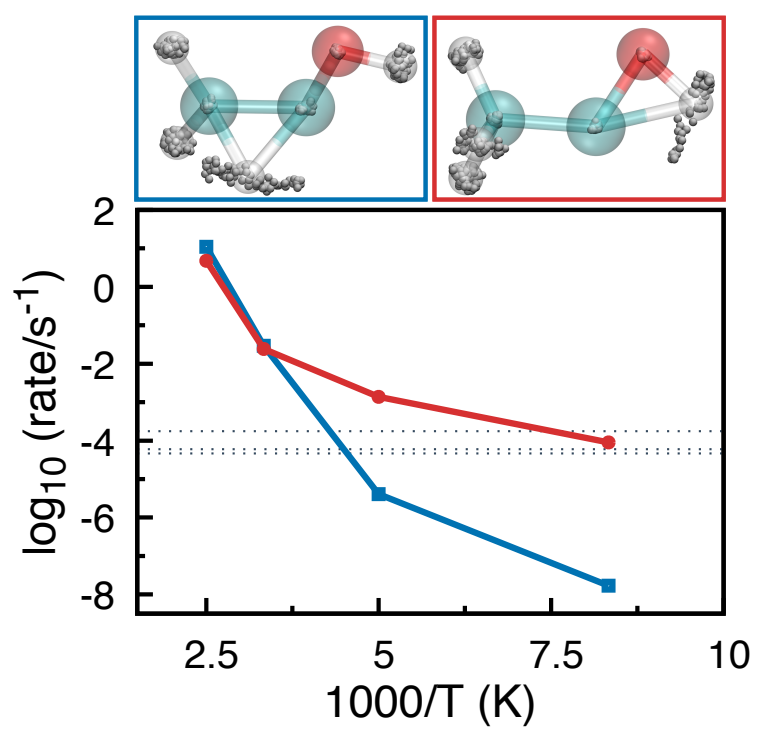

Figure 4: Temperature-dependence of the rate constants for pathway A (blue) and pathway B (red). Note that 10-base logarithm is used for plotting $\log k$. Three horizontal black dotted lines present experimentally measured rates. ${ }^{24}$ The top panels shows the typical ring polymer configurations of pathways $\mathrm{A}$ and $\mathrm{B}$, respectively.

Under lower temperatures, the quantum free energy barrier for both pathways decrease. However, as shown in Fig. 1, the barrier of the pathway B drops faster than that of pathway A. As a result, tunneling effects reduce the effective free energy barrier of pathway B more significantly. Given the fact that $\kappa\left(t_{\mathrm{p}}\right)$ of both pathways are in the same magnitude at the same temperature (see Fig. 3C and Fig. S1B in the Supporting Information), the preferred pathway will be reverted to pathway B at low temperatures.

Our theoretical results of the rate constant at a very low temperature $T=120 \mathrm{~K}$ agree with the experimental observations that acetaldehyde is the preferred product, which are measured under $T=11 \mathrm{~K}$ within three different hosting matrix (Ar, $\mathrm{Kr}$, and $\mathrm{Xe}$ ), with the corresponding rate constants (black dashed lines) presented in Fig. 4. Although we are comparing our $120 \mathrm{~K}$ results with the experimental data at $11 \mathrm{~K}, T=120 \mathrm{~K}$ is already far below $\frac{1}{2} T_{\mathrm{c}}$, half of the crossover temperatures of both pathways. This means that the thermally induced contribution to the reaction rate is minimal and going further lower temperature will not change the reaction rate, hence the $\log k$ should plateau as a function of $1 / T$. This is why our numerical results have already shown the trend of approaching the plateau value in Fig. 4. Interestingly, the ratio of the two rate constants in the plateau region of deep tunneling from out calculation is $\log k_{\mathrm{B}} / k_{\mathrm{A}}=3.7$, in a good agreement with the experimentally ${ }^{24}$ measured value 3.3 under $T=11$ K.

Simulating lower temperatures results $(T<120$ $\mathrm{K})$ requires even more beads $(n>64)$ in the ring polymer and consequently, more computational resources. In the future, this can be addressed by incorporating the ring polymer contraction scheme, ${ }^{46,47}$ where the full ring polymer beads potential $V$ is evaluated with some lower level electronic structure calculations (such as the Density Functional based Tight Binding) as a "reference" system, while a contracted ring polymer is evaluated with the higher level theory (such as KS-DFT), hence saving a huge amount of computational efforts, as demonstrated in the recent state-of-the-art AIMD-RPMD simulation of water. ${ }^{46,47}$ Another potential challenge arises from the fact that the centroid coordinate become less optimal under the low temperature, resulting in a keep decreasing $\kappa(t)$ which requires a large amount of trajectories to converge. This can be potentially addressed by using the knowledge from other non-centroid modes into the collective coordinate. ${ }^{30,31,44}$

In conclusion, we have reported, to the best of our knowledge, the first ab-initio on-the-fly RPMD rate constant calculation. We use it to investigate tunneling controlled reactions in methylhydroxycarbene. We computed the free energy profiles and reaction rate constants of two hydrogen transfer pathways in Methylhydroxycarbene. Our results suggest that below crossover temperature the intrinsic quantum tunneling effects can facilitate the chemical reaction in a pathway that is neither favored thermodynamically nor kinetically, opening up new possibilities to enable chemical transformations. Further, our ab-initio RPMD rate constant calculations provides accurate rate constant of the reaction that are in an excellent agreement with the experimental measurements. ${ }^{24}$ We demonstrated that RPMD can be conveniently combined with 
the $a b$-initio on-the-fly simulations to investigate a realistic hydrogen atom transfer system under the tunneling controlled reaction regime. This work provides an interesting and important example of using ab-initio RPMD to investigate reactions dominated by quantum tunneling effects ${ }^{26}$ to provide detailed mechanistic insights.

Theoretical Approaches. All simulations, including the free energy profile and transmission coefficient calculations are performed with an in-house modified version of the $\mathrm{CPMD}^{48}$ package version 3.15.3. The molecule is simulated with the BLYP functional ${ }^{37-39}$ in its singlet electronic ground state. The molecule is placed in an isolated simulation box of $8 \AA$ which is treated with Martyna-Tuckerman formalism, ${ }^{49}$ and using a plane wave basis with a cutoff of $80 \mathrm{Ry}$. The core electrons were treated with Troullier-Martins pseudopotentials. ${ }^{50}$ The normal mode representation is used to propagate the trajectories in all simulations. ${ }^{51}$

To characterize the progress of the reaction, we use the following reaction coordinate

$$
\xi(\mathbf{q})=\frac{\mathbf{R}_{\mathrm{DH}} \cdot \mathbf{R}_{\mathrm{DA}}}{\left|\mathbf{R}_{\mathrm{DA}}\right|}=\frac{\left(\mathbf{q}_{\mathrm{H}}-\mathbf{q}_{\mathrm{D}}\right) \cdot\left(\mathbf{q}_{\mathrm{A}}-\mathbf{q}_{\mathrm{D}}\right)}{\left|\mathbf{q}_{\mathrm{A}}-\mathbf{q}_{\mathrm{D}}\right|},
$$

where $\mathrm{H}, \mathrm{D}$ and $\mathrm{A}$ denote the transferring hydrogen atom, the donor atom, and the acceptor atom, respectively. This reaction coordinate ${ }^{20}$ measures the length of the projection of vector $\mathbf{R}_{\mathrm{DH}}$ onto the axis that connect the hydrogen donor and acceptor atoms. The larger the reaction coordinate is, the closer the transferring hydrogen to the acceptor atom. For the RPMD simulation, the centroid coordinate $\overline{\mathbf{q}}=\frac{1}{n} \sum_{j} \mathbf{q}_{j}$ is used in the above expression.

The potential of mean-force $(\mathrm{PMF}) F(\xi)$ at reaction coordinate $\xi^{i}$ is defined as

$F\left(\xi^{i}\right)=-\frac{1}{\beta} \ln \left[\frac{C_{\mathcal{N}}}{Q(N, V, T)} \int d \mathbf{p} d \mathbf{q} e^{-\beta H_{n}(\mathbf{p}, \mathbf{q})} \delta\left(\xi-\xi^{i}\right)\right]$,

where $H_{n}(\mathbf{p}, \mathbf{q})$ is the ring polymer Hamiltonian defined in Eq. 1, $C_{\mathcal{N}}=\frac{1}{(\mathcal{N} / 3) ! h^{\mathcal{N}}}$, and $Q(N, V, T)=$ $\int d \mathbf{p} d \mathbf{q} e^{-\beta H_{n}(\mathbf{p}, \mathbf{q})}$ is the ring polymer canonical partition function. The PMF is computed using the Path integral Car-Parrinello molecular dynamics (CPMD) simulations. The fictitious electron mass $m=400$ a.u. is used in the CPMD-PIMD simulation. A massive NoséHoover chain thermostat ${ }^{52}$ on every DOF was used to maintain a NVT ensemble in the simulation box. The path-integral molecular dynamics (PIMD) ${ }^{53}$ propagation is used for the nuclei, using the fictitious nuclear masses (Parrinello-Rahman mass) to be four times of the physical masses to facilitate the configurations sampling. We have carefully checked that the CPMD generated PMF is identical to the Born-Oppenheimer MD generated PMF for both the classical dynamics and the PIMD dynamics, with results provided in the Supporting Information. Since the free energy barriers for both pathways are much higher compared to thermal fluctuations, ${ }^{24}$ the blue moon ensemble approach ${ }^{41-43}$ (an enhanced sampling technique) is used to facilitate the free energy calculations in the canonical (NVT) ensemble. The details of the blue moon ensemble simulation is provided in the Supporting Information. A set of 40 constrained MD simulations was perform along the reaction coordinate from the reactant to the product in NVT ensemble. The time step was set to be 0.072 fs. Each trajectory was then equilibrated for at least 0.5 $\mathrm{ps}$, followed with a production run of at least $1 \mathrm{ps}$. The number of beads used for nuclei quantization at various temperatures is listed in Table 1, where we have carefully checked the bead-convergence, with details provided in the Supporting Information.

Table 1: The number of beads used at different temperatures in our simulations.

\begin{tabular}{ccc}
\hline \hline$T(\mathrm{~K})$ & $400-300$ & $200-120$ \\
\hline$n$ & 16 & 64 \\
\hline \hline
\end{tabular}

The ring polymer molecular dynamics transition state theory (RPMD-TST) rate constant $k_{\mathrm{TST}}$ accounts the rate component purely dictated by quantum statistics (quantum free energy barrier height). It has been shown that $k_{\text {TST }}$ coincide with the quantum mechanical TST rate theory, ${ }^{54}$ explaining the success of the RPMD rate theory. The RPMD-TST rate $k_{\mathrm{TST}}$ is expressed as

$$
k_{\mathrm{TST}}=\frac{1}{2 \pi \beta}\left\langle g_{\xi}\right\rangle_{\mathrm{c}} \frac{e^{-\beta \Delta F\left(\xi^{\ddagger}\right)}}{\int_{-\infty}^{\xi^{\ddagger}} e^{-\beta \Delta F(\xi)} d \xi},
$$

where $\xi(\overline{\mathbf{q}})$ denotes the centroid reaction coordinate (defined in Eq. 4), $\xi^{\ddagger}$ denotes the value of the dividing surface along the reaction coordinate, which is obtained at the highest value of $F(\xi(\overline{\mathbf{q}}))$ along $\xi(\overline{\mathbf{q}})$, and $\Delta F\left(\xi^{\ddagger}\right)$ represents the free energy barrier height from the bottom of the reactant well to the top of the free energy barrier at $\xi=\xi^{\ddagger}$. Further, $g_{\xi}(\overline{\mathbf{q}})=\sqrt{\sum_{i=1}^{\mathcal{N}} \frac{1}{M_{i}}\left(\frac{\partial \xi(\overline{\mathbf{q}})}{\partial \bar{q}_{i}}\right)^{2}}$, where $i$ is the index of the nuclear DOF, $M_{i}$ is the corresponding mass, and $\mathcal{N}$ is the total number of DOF. This quantity serves as the square root of the inverse reduced mass of the reaction coordinate.

To compute $\kappa(t)$ in Eq. 3, one needs to define the following side operator $h$, which is a Heaviside function of the reaction coordinate $\xi$ defined as

$$
h\left[\xi\left(\overline{\mathbf{q}}_{\mathrm{t}}\right)\right]=\left\{\begin{array}{l}
1, \text { if } \xi \geqslant \xi^{\ddagger} \\
0, \text { if } \xi<\xi^{\ddagger} .
\end{array}\right.
$$

The flux operator is the time derivative of the side operator, expressed as

$$
\mathcal{F}\left(\overline{\mathbf{q}}_{0}, \overline{\mathbf{p}}_{0}\right)=\dot{h}\left[\xi\left(\overline{\mathbf{q}}_{0}\right)\right]=\sum_{i=1}^{\mathcal{N}} \delta\left(\xi-\xi^{\ddagger}\right) \frac{d \xi}{d \bar{q}_{i}} \bar{p}_{i} .
$$


In the $\kappa(t)$ simulations, one needs to generate the constraint canonical ensemble on the dividing surface $\xi=$ $\xi^{\ddagger}$. These configurations are generated by constraining the ring polymer reaction coordinate $\xi(\overline{\mathbf{q}})$ on the dividing surface $\xi^{\ddagger}(\overline{\mathbf{q}})$, through the SHAKE algorithm as implemented in the CPMD package. ${ }^{55,56}$ A sampling trajectory with the CPMD-PIMD simulation is used to generate the constrained canonical configuration, with each constraint geometries picked at every $0.36 \mathrm{ps}$ from the sampling trajectory.

The transmission coefficient $\kappa$ is calculated using RPMD trajectories that are released from the dividing surface at $\xi(\overline{\mathbf{q}})$, and the dynamics is propagated using RPMD in the normal mode representation, with the physical masses of the nuclei the NVE ensemble (without using any thermostat). Due to the fact that the highest normal mode frequency $\omega_{n}=n / \beta \hbar$ in RPMD will be close to the fictitious electronic frequencies in CPMD, the adiabaticity condition in CPMD method is no longer valid. As a result, all the trajectories for computing $\kappa(t)$ are simulated with Born-Oppenheimer molecular dynamics (BOMD) as implemented in CPMD package. Between 500 and 1200 trajectories are released from the dividing surfaces, each RPMD trajectory is evolved for 100 fs (during which the flux-side correlation function is guaranteed to reach the plateau value) with a time step of 0.24 fs and with the initial velocities sampled from the Maxwell-Boltzmann distribution.

\section{Supporting Information}

The Supporting Information is available free of charge via the Internet at http://pubs.acs.org.

Computational Details, Additional Results for Reaction Pathway A, Choice of the Density Functional, Tests of Bead Convergence, Free Energy Profiles Computed from CPMD Versus BOMD.

\section{Acknowledgement}

This work was supported by the National Science Foundation CAREER Award under Grant No. CHE1845747. Computing resources were provided by the Center for Integrated Research Computing (CIRC) at the University of Rochester, XSEDE under a grant CHE-190016.

\section{References}

(1) Markland, T. E.; Ceriotti, M. Nuclear quantum effects enter the mainstream. Nat. Rev. Chem. 2018, 2, 0109.

(2) Hammes-Schiffer, S. Quantum effects in complex systems: summarizing remarks. Faraday Discuss. 2020, 221, 582-588.

(3) Kawashima, Y.; Tachikawa, M. Nuclear quantum effect on intramolecular hydrogen bond of hydrogen maleate anion: An ab initio path integral molecular dynamics study. Chem. Phys. Lett. 2013, 571, 23-27.

(4) Fang, W.; Chen, J.; Rossi, M.; Feng, Y.; Li, X.-Z.; Michaelides, A. Inverse Temperature Dependence of Nuclear Quantum Effects in DNA Base Pairs. J. Phys. Chem. Lett. 2016, 7, 2125-2131.

(5) Litman, Y.; Richardson, J. O.; Kumagai, T.; Rossi, M. Elucidating the Nuclear Quantum Dynamics of Intramolecular Double Hydrogen Transfer in Porphycene. J. Am. Chem. Soc. 2019, 141, 2526-2534.

(6) Pérez, A.; Tuckerman, M. E.; Hjalmarson, H. P.; von Lilienfeld, O. A. Enol tautomers of WatsonCrick base pair models are metastable because of nuclear quantum effects. J. Am. Chem. Soc. 2010, 132, 11510-11515.

(7) Ceriotti, M.; Fang, W.; Kusalik, P. G.; McKenzie, R. H.; Michaelides, A.; Morales, M. A.; Markland, T. E. Nuclear Quantum Effects in Water and Aqueous Systems: Experiment, Theory, and Current Challenges. Chem. Rev. 2016, 116, 75297550 .

(8) Hammes-Schiffer, S.; Tully, J. C. Proton transfer in solution: Molecular dynamics with quantum transitions. J. Chem. Phys. 1994, 101, 4657-4667.

(9) Roberts, S. T.; Ramasesha, K.; Petersen, P. B.; Mandal, A.; Tokmakoff, A. Proton transfer in concentrated aqueous hydroxide visualized using ultrafast infrared spectroscopy. J. Phys. Chem. A 2011, 115, 3957-3972.

(10) Hammes-Schiffer, S.; Tully, J. C. Proton transfer in solution: Molecular dynamics with quantum transitions. J. Chem. Phys. 1994, 101, 4657-4667.

(11) Collepardo-Guevara, R.; Craig, I. R.; Manolopoulos, D. E. Proton transfer in a polar solvent from ring polymer reaction rate theory. J. Chem. Phys. 2008, 128, 144502-144514. 
(12) Boekelheide, N.; Salomón-Ferrer, R.; Miller III, T. F. Dynamics and dissipation in enzyme catalysis. Proc. Natl. Acad. Sci. U.S.A. 2011, 108, 16159-16163.

(13) Suleimanov, Y. V.; Collepardo-Guevara, R.; Manolopoulos, D. E. Bimolecular reaction rates from ring polymer molecular dynamics: application to $\mathrm{H}+\mathrm{CH}_{4} \rightarrow \mathrm{H}_{2}+\mathrm{CH}_{3}$. J. Chem. Phys. 2011, 134, 044131-044140.

(14) Kretchmer, J. S.; Miller, T. F. Direct simulation of proton-coupled electron transfer across multiple regimes. J. Chem. Phys. 2013, 138, 134109 134128.

(15) Hammes-Schiffer, S.; Soudackov, A. V. Protoncoupled electron transfer in solution, proteins, and electrochemistry. J. Phys. Chem. B 2008, 112, 14108-14123.

(16) Hazra, A.; Soudackov, A. V.; Hammes-Schiffer, S. Role of solvent dynamics in ultrafast photoinduced proton-coupled electron transfer reactions in solution. J. Phys. Chem. B 2010, 114, 12319-12332.

(17) Hazra, A.; Soudackov, A. V.; Hammes-Schiffer, S. Isotope Effects on the Nonequilibrium Dynamics of Ultrafast Photoinduced Proton-Coupled Electron Transfer Reactions in Solution. J. Phys. Chem. Lett. 2011, 2, 36-40.

(18) Benkovic, S. J.; Hammes-Schiffer, S. A Perspective on Enzyme Catalysis. Science 2003, 301, 11961202 .

(19) Zhang, C.; Michaelides, A. Quantum nuclear effects on the location of hydrogen above and below the palladium (100) surface. Surf. Sci. 2011, 605, 689-694.

(20) Cendagorta, J. R.; Powers, A.; Hele, T. J. H.; Marsalek, O.; Bačić, Z.; Tuckerman, M. E. Competing quantum effects in the free energy profiles and diffusion rates of hydrogen and deuterium molecules through clathrate hydrates. Phys. Chem. Chem. Phys. 2016, 18, 32169-32177.

(21) McIntosh, E. M.; Wikfeldt, K. T.; Ellis, J.; Michaelides, A.; Allison, W. Quantum Effects in the Diffusion of Hydrogen on $\mathrm{Ru}(0001)$. J. Phys. Chem. Lett. 2013, 4, 1565-1569.

(22) Hund, F. Zur Deutung der Molekelspektren. III. Zeitschrift für Physik 1927, 43, 805-826.

(23) Wigner, E. On the Quantum Correction For Thermodynamic Equilibrium. Phys. Rev. 1932, 40, 749-759.
(24) Schreiner, P. R.; Reisenauer, H. P.; Ley, D.; Gerbig, D.; Wu, C.-H.; Allen, W. D. Methylhydroxycarbene: Tunneling Control of a Chemical Reaction. Science 2011, 332, 1300-1303.

(25) Ley, D.; Gerbig, D.; Wagner, J. P.; Reisenauer, H. P.; Schreiner, P. R. Cyclopropylhydroxycarbene. J. Am. Chem. Soc. 2011, 133, 1361413621.

(26) Schreiner, P. R. Tunneling Control of Chemical Reactions: The Third Reactivity Paradigm. J. Am. Chem. Soc. 2017, 139, 15276-15283.

(27) Kästner, J. Path length determines the tunneling decay of substituted carbenes. Chem. Eur. J. 2013, 19, 8207-8212.

(28) Habershon, S.; Manolopoulos, D. E.; Markland, T. E.; Miller, T. F. Ring-Polymer Molecular Dynamics: Quantum Effects in Chemical Dynamics from Classical Trajectories in an Extended Phase Space. Annu. Rev. Phys. Chem. 2013, 64, 387-413.

(29) Craig, I. R.; Manolopoulos, D. E. Quantum statistics and classical mechanics: Real time correlation functions from ring polymer molecular dynamics. J. Chem. Phys. 2004, 121, 3368-3373.

(30) Craig, I. R.; Manolopoulos, D. E. Chemical reaction rates from ring polymer molecular dynamics. J. Chem. Phys. 2005, 122, 084106-084117.

(31) Craig, I. R.; Manolopoulos, D. E. A refined ring polymer molecular dynamics theory of chemical reaction rates. J. Chem. Phys. 2005, 123, 034102034111.

(32) Feynman, R.; Hibbs, A. R. Quantum Mechanics and Path Integrals; McGraw-Hill, 1965.

(33) Hele, T. J. H.; Willatt, M. J.; Muolo, A.; Althorpe, S. C. Boltzmann-conserving classical dynamics in quantum time-correlation functions: "Matsubara dynamics". J. Chem. Phys. 2015, 142, 134103.

(34) Hele, T. J. H.; Willatt, M. J.; Muolo, A.; Althorpe, S. C. Communication: Relation of centroid molecular dynamics and ring-polymer molecular dynamics to exact quantum dynamics. J. Chem. Phys. 2015, 142, 191101.

(35) Chandler, D.; Wolynes, P. G. Exploiting the isomorphism between quantum theory and classical statistical mechanics of polyatomic fluids. $J$. Chem. Phys. 1981, 74, 4078-4095. 
(36) Menzeleev, A. R.; Ananth, N.; Miller, T. F. Direct simulation of electron transfer using ring polymer molecular dynamics: comparison with semiclassical instanton theory and exact quantum methods. J. Chem. Phys. 2011, 135, 074106-074122.

(37) Becke, A. D. Density-functional exchange-energy approximation with correct asymptotic behavior. Phys. Rev. A 1988, 38, 3098-3100.

(38) Colle, R.; Salvetti, O. Approximate calculation of the correlation energy for the closed shells. Theor. Chim. Acta 1975, 37, 329-334.

(39) Lee, C.; Yang, W.; Parr, R. G. Development of the Colle-Salvetti correlation-energy formula into a functional of the electron density. Phys. Rev. B 1988, 37, 785-789.

(40) Frenkel, D.; Smit, B. Understanding Molecular Simulation; Elsevier, 2002.

(41) Carter, E. A.; Ciccotti, G.; Hynes, J. T.; Kapral, R. Constrained reaction coordinate dynamics for the simulation of rare events. Chem. Phys. Lett. 1989, 156, 472-477.

(42) Sprik, M.; Ciccotti, G. Free energy from constrained molecular dynamics. J. Chem. Phys. 1998, 109, 7737-7744.

(43) Laria, D.; Ciccotti, G.; Ferrario, M.; Kapral, R. Activation free energy for proton transfer in solution. Chem. Phys. 1994, 180, 181-189.

(44) Richardson, J. O.; Althorpe, S. C. Ring-polymer molecular dynamics rate-theory in the deeptunneling regime: Connection with semiclassical instanton theory. J. Chem. Phys. 2009, 131, 214106-214117.

(45) Zhang, Y.; Stecher, T.; Cvitaš, M. T.; Althorpe, S. C. Which Is Better at Predicting Quantum-Tunneling Rates: Quantum TransitionState Theory or Free-Energy Instanton Theory? J. Phys. Chem. Lett. 2014, 5, 3976-80.

(46) Markland, T. E.; Manolopoulos, D. E. An efficient ring polymer contraction scheme for imaginary time path integral simulations. J. Chem. Phys. 2008, 129, 024105-024114.

(47) Marsalek, O.; Markland, T. E. Ab initio molecular dynamics with nuclear quantum effects at classical cost: Ring polymer contraction for density functional theory. J. Chem. Phys. 2016, 144, 054112 054122 .

(48) Hutter, J.; Alavi, A.; Deutsch, T.; Bernasconi, M.; Goedecker, S.; Marx, D.; Tuckerman, M.; Parrinello, M. CPMD: Car-Parinello Molecular Dynamics, version 3.15.3. IBM Corp 1990-2008 and
MPI für Festkörperforschung Stuttgart 19972001. http://www.cpmd.org.

(49) Martyna, G. J.; Tuckerman, M. E. A reciprocal space based method for treating long range interactions in ab initio and forcefield-based calculations in clusters. J. Chem. Phys. 1999, 110, 28102821.

(50) Troullier, N.; Martins, J. L. Efficient pseudopotentials for plane-wave calculations. Phys. Rev. B 1991, 43, 1993-2006.

(51) Berne, B. J.; Thirumalai, D. On the Simulation of Quantum Systems: Path Integral Methods. Annu. Rev. Phys. Chem. 1986, 37, 401-424.

(52) Martyna, G. J.; Klein, M. L.; Tuckerman, M. E. Nosé-Hoover chains: The canonical ensemble via continuous dynamics. J. Chem. Phys. 1992, 97, 2635-2643.

(53) Tuckerman, M. E.; Marx, D.; Klein, M. L.; Parrinelle, M. Efficient and general algorithms for path integral Car-Parrinello molecular dynamics. J. Chem. Phys. 1996, 104, 5579-5588.

(54) Hele, T. J. H.; Althorpe, S. C. Derivation of a true $\left(\mathrm{t} \rightarrow 0^{+}\right)$quantum transition-state theory. I. Uniqueness and equivalence to ring-polymer molecular dynamics transition-state-theory. $J$. Chem. Phys. 2013, 138, 084108-084120.

(55) Ryckaert, J.-P.; Ciccotti, G.; Berendsen, H. J. Numerical integration of the cartesian equations of motion of a system with constraints: molecular dynamics of n-alkanes. J. Comput. Phys. 1977, 23, 327-341.

(56) Tuckerman, M. E.; Parrinello, M. Integrating the Car-Parrinello equations. II. Multiple time scale techniques. J. Chem. Phys. 1994, 101, 1316-1329. 